\title{
Hämatologische Neoplasien: Therapie im Wandel
}

\author{
Klinischer Benefit durch den Immunmodulator Lenalidomid
}

Für die Zweitlinientherapie des Multiplen Myeloms in Kombination mit Dexamethason ist Lenalidomid zugelassen, die Substanz wird derzeit aber auch bei einer Reihe anderer hämatologischer Entitäten getestet. Viel versprechende Ergebnisse konnten bereits in der Behandlung von CLL und NHL erzielt werden. Ein umfassendes Studienprogramm prüft Lenalidomid in verschiedenen Settings, etwa als Erstlinientherapie des Multiplen Myeloms sowie im Rahmen von Konsolidierungsund Erhaltungskonzepten. Ein aktueller Ausblick wurde bei der Frühjahrstagung der Österreichischen Gesellschaft für Hämatologie und Onkologie im vergangenen April in Bregenz gegeben.

„Die Substanz wirkt auf Tumorzellen und deren Mikroenvironment", erläuterte Univ.-Prof. Dr. Richard Greil, Univ.-Klinik für Innere Medizin III, Paracelsus Medizinische Privatuniversität, Salzburg. Eine Tumorzell-Zytotoxizität resultiert aus verschiedenen Effekten, etwa einer Proliferation der T-Zellen und einer Steigerung von Funktion und Zahl der NK-Zellen. Greil: „Lenalidomid hemmt die Angiogenese in vitro und wirkt insbesondere auch durch eine Wiederherstellung der dysregulierten immunologischen Synapsenformation der T-Zellen.“

Zwei frühe Phase-II-Studien belegen ein gutes Ansprechen auf eine LenalidomidMonotherapie bei weit fortgeschrittener bzw. relapsierter CLL mit Gesamtansprechraten (ORR) in der Höhe von 32 bzw. 47 Prozent $(1,2)$. „Auch Patienten mit prognostisch ungünstigen Parametern wie 11q-oder $17 \mathrm{p}$-Deletionen sprachen in den Studien gut auf Lenalidomid an", betonte Greil (Tab. 1). Das mediane progressionsfreie Überleben (PFS) war in dieser Hochrisiko-

\section{LITERATUR}

1 Chanan-Khan A et al. (2006) J Clin Oncol 24: 5343-5349

2 Ferrajoli A et al. (2008) Blood 111: 5291-5297

3 Sher T et al. (2010) Leukemia \& Lymphoma

51(1): 85-88

4 Egle A et al. (2009) ASH, Abstract 3453

5 Witzig TE et al. (2009) ASH, Abstract 1676

\begin{tabular}{|c|c|c|c|}
\hline & $\mathbf{n}$ & ORR & Ansprechen \\
\hline $\operatorname{Del}(17)(p 13.1)$ & 6 & $50 \%$ & 3PR, 1SD \\
\hline Del(11)(q22.23) & 10 & $40 \%$ & 1CR, 3PR, 4SD \\
\hline Fludarabin-refraktär & 23 & $30 \%$ & 1CR, 6PR, 3SD \\
\hline ZAP-70+ & 10 & $60 \%$ & 4CR, 2PR, 2SD \\
\hline \multicolumn{4}{|l|}{ nach Chanan-Khan et al. 2006} \\
\hline & $\mathbf{n}$ & ORR & Ansprechen \\
\hline $\operatorname{Del}(17)(p 13.1)$ & 8 & $13 \%$ & 1PR, 2SD \\
\hline $\operatorname{Del}(11)(q 22.23)$ & 18 & $39 \%$ & 2CR, 4PR, 5SD(1) \\
\hline Unmutiertes IgVH & 29 & $24 \%$ & 2CR, 5PR, 8SD \\
\hline Fludarabin-refraktär & 12 & $25 \%$ & 3PR, 4SD \\
\hline$-70+$ & 17 & $24 \%$ & 1CR, 3PR, 3SD \\
\hline
\end{tabular}

wie vor groß. In den vergangenen Jahren konnten Studien die Wirksamkeit von Lenalidomid bei unterschiedlichen NHL-Subtypen demonstrieren. Im Rahmen der großen Phase-II-Studie NHL-003 erhielten 217 stark vorbehandelte Patienten mit rezidiviertem/refraktärem aggressivem NHL eine Monotherapie mit Lenalidomid (5). „Ein Ansprechen wurde in allen histologischen Subgruppen beobachtet", erklärte Univ.-Prof. Dr. Johannes Drach, Univ.-Klinik für Innere Medizin I, Medizinische Universität Wien. „Lenalidomid zeigt bei einem günstigen Sicherheitsprofil Effektivität in der Behandlung aller NHL-Subtypen“, resümierte Drach die aktuellen Studienergebnisse.
Klientel mit 12,1 Monaten beachtlich (3). Ein Vergleich von Lenalidomid mit anderen Monotherapien bei fortgeschrittener bzw. relapsierter CLL zeigt ähnliche Ansprech- und Remissionsraten.

\section{Management von Tumor-Flare und Tumorlyse}

Als hämatologische Nebenwirkungen manifestieren sich unter Lenalidomid hauptsächlich höher gradige Thrombozytopenien und Neutropenien, die aber in der Regel gut behandelbar sind. Im Rahmen der Therapie einer B-CLL wurden in frühen Studien schwere Tumorlyse- und Tumor-Flare-Reaktionen beobachtet. Lymphknotenschwellungen, Hautausschläge, niedriges Fieber und Knochenschmerzen kennzeichnen den Tumor-Flare, der oft am Tag zwei bis vier der Therapie auftritt und etwa 14 Tage anhält. „Die Symptomatik muss von einer Krankheitsprogression abgegrenzt werden“, hob Greil hervor. Je nach Schweregrad erfolgt die Therapie mit NSAR, Kortikosteroiden und/ oder narkotischen Analgetika. Das Management des Tumorlysesyndroms stützt sich auf ausreichende Hydratation und die prophylaktische Gabe von Allopurinol.

\section{Breites NHL-Wirkspektrum}

Bei Non-Hodgkin-Lymphom (NHL) ist der Bedarf an weiteren Therapieoptionen nach

\section{MM: Eignung für die Langzeitanwendung}

Das Multiple Myelom (MM) ist durch eine hohe Rezidivneigung gekennzeichnet, die auf dem neuerlichen Wachstum verbliebener Tumorzellen und einer Immunsuppression basiert. Dementsprechend sollten Therapien an diesen beiden Komponenten ansetzen. Lenalidomid beeinflusst die Krankheitsmechanismen bei MM auf vielfältige Weise. „Die tumoriziden und immunmodulierenden Effekte führen zur Erreichung dauerhafter Remissionen", bezog sich Univ.-Prof. Dr. Heinz Ludwig, 1. Medizinische Abteilung, Wilhelminenspital Wien, auf die duale Aktivität der Substanz. „Eine kontinuierliche Behandlung kann das klinische Ergebnis weiter verbessern und wird bei relapsiertem bzw. refraktärem MM empfohlen." Aufgrund des Fehlens kumulativer Nebenwirkungen eignet sich der Immunmodulator speziell für den Langzeiteinsatz.

„MPR-R bildet bei älteren Patienten mit MM-Erstdiagnose eine neue Standardoption," stellte Ludwig aufrgund der Studienergebnisse fest.

Dr. Judith Moser

Quelle: Frühjahrstagung der ÖGHO, 9. April 2010, Bregenz 